\title{
Searches for direct supersymmetric gaugino and slepton pair production in final states with leptons with the ATLAS detector
}

\author{
Anders Floderus ${ }^{1, a}$ on behalf of the ATLAS Collaboration \\ ${ }^{1}$ Lund University, Sweden
}

\begin{abstract}
A search for direct production of supersymmetric gauginos and sleptons in final states with two and three leptons is presented. The two lepton analysis uses $4.7 \mathrm{fb}^{-1}$ of proton-proton collision data at $\sqrt{s}=7 \mathrm{TeV}$, while the three lepton analysis uses $13 \mathrm{fb}^{-1}$ at $\sqrt{s}=8 \mathrm{TeV}$. All data were recorded by the ATLAS detector at the Large Hadron Collider. The results are interpreted in the phenomenological minimal supersymmetric Standard Model, as well as simplified models. No significant excess above the Standard Model expectation is observed. Limits on the particle masses are placed at the $95 \%$ confidence level. In simplified models, chargino masses are excluded up to $580 \mathrm{GeV}$.
\end{abstract}

\section{Introduction}

Supersymmetry (SUSY) [1-9] postulates that each known particle has a partner whose spin differs by one-half unit with respect to its Standard Model (SM) counterpart. Conservation of R-parity [10-14] implies that SUSY particles can only be produced in pairs, and must eventually decay into some combination of SM particles and the lightest SUSY particle (LSP), which is stable.

Charginos $\left(\tilde{\chi}_{1}^{ \pm}, \tilde{\chi}_{2}^{ \pm}\right)$and neutralinos $\left(\tilde{\chi}_{1}^{0}, \tilde{\chi}_{2}^{0}, \tilde{\chi}_{3}^{0}, \tilde{\chi}_{4}^{0}\right)$, collectively known as gauginos, are the mass eigenstates formed from the linear superposition of the SUSY partners of the Higgs and electroweak gauge bosons. The SUSY partners of the gluons, quarks and leptons are called gluinos $(\tilde{g})$, squarks $(\tilde{q})$ and sleptons $(\tilde{\ell})$. Gauginos and sleptons may dominate SUSY production at the LHC if they are light compared to gluinos and squarks.

A search for direct production of supersymmetric gauginos and sleptons in final states with leptons (electrons or muons) using the ATLAS [15] detector is presented. It is divided into two separate analyses, which are optimized independently. The two lepton analysis looks for two lepton final states, and the three lepton analysis looks for three lepton final states.

\section{New Physics Scenarios}

The results are interpreted in the framework of the phenomenological minimal supersymmetric Standard Model (pMSSM) [16]. In these models, $\tan \beta$ is set to 6 . The gluinos, squarks and left-handed sleptons are taken to be heavy with masses greater than $2 \mathrm{TeV}$, and the righthanded sleptons are assumed to be degenerate with $m_{\tilde{\ell}_{R}}=$ $\left(m_{\tilde{\chi}_{2}^{0}}+m_{\tilde{\chi}_{1}^{0}}\right) / 2$. Signal grids are then generated in the $M_{2}-\mu$

\footnotetext{
a e-mail: anders.savonen.floderus@cern.ch
}

plane for three different values of $M_{1}$, where $M_{1}$ and $M_{2}$ are the gaugino masses and $\mu$ is the Higgs mass parameter. In a pMSSM variant referred to as the direct slepton model, left-handed sleptons are lighter with $m_{\tilde{\ell}_{L}}=m_{\tilde{\ell}_{R}}$, and all gauginos masses except $\tilde{\chi}_{1}^{0}$ are set to $2.5 \mathrm{TeV}$ to favor slepton production.

The results are also interpreted in simplified models [17] where the only free parameters are the masses of the relevant particles, namely $\tilde{\chi}_{1}^{0}, \tilde{v}, \tilde{\ell}_{L}, \tilde{\chi}_{1}^{ \pm}$and $\tilde{\chi}_{2}^{0}$. Righthanded charged sleptons are very heavy with masses above $100 \mathrm{TeV}$. Two scenarios, both with $m_{\tilde{\chi}_{2}^{0}}=m_{\tilde{\chi}_{1}^{ \pm}}$, are considered. The first scenario includes intermediate sleptons with $m_{\tilde{v}}=m_{\tilde{\ell}_{L}}=\left(m_{\tilde{\chi}_{2}^{0}}+m_{\tilde{\chi}_{1}^{0}}\right) / 2$. In the second scenario, all slepton masses are above $100 \mathrm{TeV}$. Signal grids are generated in the $\tilde{\chi}_{1}^{ \pm}-\tilde{\chi}_{1}^{0}$ plane.

\section{Two Lepton Analysis}

The two lepton analysis [18] uses $4.7 \mathrm{fb}^{-1}$ of proton-proton collision data recorded by the ATLAS detector at $\sqrt{\mathrm{s}}=$ $7 \mathrm{TeV}$. The event selection, signal regions, background evaluation method and results are presented.

\subsection{Event Selection}

Events are selected using a combination of single and double lepton triggers. Each reconstructed triggering lepton must be matched to an online lepton. Jet candidates are reconstructed using the anti- $k_{t}$ jet algorithm [19] with a distance parameter of 0.4 , and are required to satisfy $p_{\mathrm{T}}>20 \mathrm{GeV}$ and $|\eta|<4.5$. Electron candidates must have $p_{\mathrm{T}}>10 \mathrm{GeV},|\eta|<2.47$, and pass "medium" [20] shower shape and track selection criteria. Muon candidates are required to have matching muon spectrometer and inner detector tracks, and to satisfy $p_{\mathrm{T}}>10 \mathrm{GeV}$ and $|\eta|<2.4$. 
A subset of the object candidates are then selected as signal objects. Signal jets must have $p_{\mathrm{T}}>30 \mathrm{GeV},|\eta|<2.5$ and a jet vertex fraction $(\mathrm{JVF})>0.75$. The JVF, which is defined as the fraction of the jet $p_{\mathrm{T}}$ that is associated to the primary vertex, is used mainly to reject pileup jets. Signal electrons and muons are required to be isolated, and the electrons must pass "tight" [20] selection criteria.

The missing transverse energy $E_{\mathrm{T}}^{\text {miss }}$ is the magnitude of the missing transverse momentum, $\mathbf{p}_{\mathrm{T}}^{\text {miss }}$. This in turn is the negative of the vector sum of the transverse momentum of all electron and muon candidates, all jets, and all calibrated calorimeter energy clusters with $|\eta|<4.9$ not associated to such objects. The two lepton analysis makes use of the relative $E_{\mathrm{T}}^{\text {miss }}$, denoted $E_{\mathrm{T}}^{\text {miss,rel. }}$. It is defined as

$$
E_{\mathrm{T}}^{\text {miss,rel. }}= \begin{cases}E_{\mathrm{T}}^{\mathrm{miss}} & \text { if } \Delta \phi_{\ell, j} \geq \pi / 2 \\ E_{\mathrm{T}}^{\mathrm{miss}} \times \sin \Delta \phi_{\ell, j} & \text { if } \Delta \phi_{\ell, j}<\pi / 2\end{cases}
$$

where $\Delta \phi_{\ell, j}$ is the azimuthal angle between the $\mathbf{p}_{\mathrm{T}}^{\text {miss }}$ and the closest signal electron, muon or jet. In events where the momentum of an object has been significantly mismeasured, such that the $\mathbf{p}_{\mathrm{T}}^{\text {miss }}$ is aligned with the object, cutting on $E_{\mathrm{T}}^{\text {miss,rel. }}$ takes into account only the $E_{\mathrm{T}}^{\text {miss }}$ component perpendicular to that object.

\subsection{Signal Regions}

The two lepton analysis uses four signal regions (SRs), which target different processes as shown in Table 1. An $m_{\ell \ell}>20 \mathrm{GeV}$ cut is common to all SRs. Their individual definitions are given in Table 2 . The main signal region, SR- $m_{\mathrm{T} 2}$, provides sensitivity to both $\tilde{\chi}_{1}^{ \pm}$and $\tilde{\ell}^{ \pm}$pair production by cutting on the $m_{\mathrm{T} 2}$ variable [21,22] defined as

$$
m_{\mathrm{T} 2}=\min _{\mathbf{q}_{\mathrm{T}}+\mathbf{r}_{\mathrm{T}}=\mathbf{p}_{\mathrm{T}}^{\text {miss }}}\left[\max \left(m_{T}\left(\mathbf{p}_{\mathrm{T}}^{\ell_{1}}, \mathbf{q}_{\mathrm{T}}\right), m_{T}\left(\mathbf{p}_{\mathrm{T}}^{\ell_{2}}, \mathbf{r}_{\mathrm{T}}\right)\right)\right]
$$

where $\mathbf{p}_{\mathrm{T}}^{\ell_{1}}$ and $\mathbf{p}_{\mathrm{T}}^{\ell_{2}}$ are the transverse momenta of the two leptons, and $\mathbf{q}_{\mathrm{T}}$ and $\mathbf{r}_{\mathrm{T}}$ are two vectors which satisfy $\mathbf{q}_{\mathrm{T}}+\mathbf{r}_{\mathrm{T}}=\mathbf{p}_{\mathrm{T}}^{\text {miss }} . m_{\mathrm{T}}$ indicates the transverse mass, $m_{\mathrm{T}}=\sqrt{2 E_{\mathrm{T}, \mathrm{p}}^{\ell_{1}} E_{\mathrm{T}, \mathrm{q}}(1-\cos \phi)}$, where $E_{\mathrm{T}}$ is the transverse energy of a particle and $\phi$ is the angle between the two particles in the transverse plane.

The SR-OSjveto region is sensitive to $\tilde{\chi}_{1}^{ \pm}$pair production, and together with SR-SSjveto it also targets three lepton final states where one lepton is either not reconstructed, or falls outside the acceptance of the detector. This provides complementarity to the three lepton analysis. SR-2jets includes a top veto based on the $m_{\mathrm{CT}}$ variable [23]. $m_{\mathrm{CT}}$ is defined as

$m_{\mathrm{CT}}\left(\chi_{1}, \chi_{2}\right)=\left[E_{t}^{2}\left(\chi_{1}\right)+E_{t}^{2}\left(\chi_{2}\right)\right]^{1 / 2}-\left[p_{T}^{2}\left(\chi_{1}\right)+p_{T}^{2}\left(\chi_{2}\right)\right]^{1 / 2}$ where $\chi_{i}$ is either a lepton or jet. Events consistent with $t \bar{t}$ kinematics, as described in [24], are rejected.

\subsection{Background Evaluation}

The main real backgrounds are $t \bar{t}$, single top, $Z / \gamma^{*}+$ jets and dibosons ( $W W, W Z$ and $Z Z$ ). Each background is estimated by defining control regions (CRs) which are dominated by the process of interest, and then extrapolating
Table 1. Process targeted by each two lepton signal region [18].

\begin{tabular}{cc}
\hline Targeted Process & Signal Region \\
\hline \multicolumn{3}{c}{ Two-lepton Final States } \\
$\tilde{\ell}^{ \pm} \tilde{\ell}^{\mp} \rightarrow\left(\ell^{\mp} \tilde{\chi}_{1}^{0}\right)+\left(\ell^{\mp} \tilde{\chi}_{1}^{0}\right)$ & SR- $m_{\mathrm{T} 2}$ \\
$\tilde{\chi}_{1}^{ \pm} \tilde{\chi}_{1}^{\mp} \rightarrow\left(\ell^{\mp} v \tilde{\chi}_{1}^{0}\right)+\left(\ell^{\mp} v \tilde{\chi}_{1}^{0}\right)$ & SR- $m_{\mathrm{T} 2}$, SR-OSjveto \\
$\tilde{\chi}_{2}^{0} \tilde{\chi}_{1}^{ \pm} \rightarrow\left(\ell^{\mp} \ell^{\mp} \tilde{\chi}_{1}^{0}\right)+\left(q \bar{q}^{\prime} \tilde{\chi}_{1}^{0}\right)$ & SR-2jets \\
\hline \multicolumn{3}{c}{ Three-lepton Final States } \\
$\tilde{\chi}_{2}^{0} \tilde{\chi}_{1}^{ \pm} \rightarrow\left(\ell^{\mp} \ell^{\mp} \tilde{\chi}_{1}^{0}\right)+\left(\ell^{\mp} v \tilde{\chi}_{1}^{0}\right)$ & SR-OSjveto, SR-SSjveto \\
\hline
\end{tabular}

Table 2. Two lepton signal region definitions. OS (SS) denotes two opposite-sign (same-sign) signal leptons, of same (SF) or different (DF) flavour. Values are in units of GeV. A $Z$-veto refers to the rejection of events with $m_{\ell \ell}$ within $10 \mathrm{GeV}$ of the $Z$ mass [18]. See text for further details.

\begin{tabular}{lcccc}
\hline SR- & $m_{\mathrm{T} 2}$ & OSjveto & SSjveto & 2jets \\
\hline charge & OS & OS & SS & OS \\
flavour & any & \multicolumn{2}{c}{ any } & SF \\
$m_{\ell \ell}$ & $Z$-veto & $Z$-veto & - & $Z$-veto \\
signal jets & $=0$ & \multicolumn{2}{c}{$=0$} & $\geq 2$ \\
signal $b$-jets & - & - & $=0$ \\
$E_{\mathrm{T}}^{\text {miss,rel. }}$ & $>40$ & $>100$ & $>50$ \\
other & $m_{\mathrm{T} 2}>90$ & - & $m_{\mathrm{CT}}$-veto \\
\hline
\end{tabular}

the number of events observed in the control regions to the signal regions. The number of events $N_{\mathrm{X}}^{\mathrm{CR}}$ in the CR is obtained from data, and the extrapolation is done with a transfer factor $\mathcal{T}$, which is obtained using Monte Carlo (MC). The number of events $N_{\mathrm{X}}^{\mathrm{SR}}$ in the $\mathrm{SR}$ is given by

$$
N_{\mathrm{X}}^{\mathrm{SR}}=N_{\mathrm{X}}^{\mathrm{CR}} \times \mathcal{T}=N_{\mathrm{X}}^{\mathrm{CR}} \times\left(\frac{N_{\mathrm{X}}^{\mathrm{SR}}}{N_{\mathrm{X}}^{\mathrm{CR}}}\right)_{\mathrm{MC}} .
$$

Control regions are defined for top, $W W$ and $Z+X$, which includes $Z / \gamma^{*}+$ jets, $W Z$ and $Z Z$. The definitions are shown in Table 3. The fake background (events containing misidentified jets and real leptons from conversions) is obtained using the matrix method, similarly to what is described in [25]. In SR-SSjveto, there is a "chargeflip" contribution, arising from electrons whose charge is mis-identified after they undergo hard bremsstrahlung with subsequent photon conversion. The charge-flip probability is measured from $Z$ events in data using a likelihood technique [26]. If a signal region or process does not have a control region listed, the background contribution is taken from MC.

\subsection{Results}

Good agreement between data and Standard Model expectation is observed in all SRs. Upper limits on the production cross-section times acceptance times efficiency $(\sigma \times \varepsilon \times A)$ of new physics are set at the $95 \%$ confidence level using the modified frequentist $\mathrm{CL}_{\mathrm{s}}$ prescription [27]. Figure 1 shows the $\tilde{\ell}^{ \pm}$pair production exclusion region in the direct slepton pMSSM. Left-handed slepton masses 
Table 3. Two lepton control region definitions. Top is estimated in all OS SRs, $W W$ in SR-OSjveto and $Z+X$ in all SF channels of the OS SRs. When the CRs use different cuts for each SR,

they are given as a comma separated list in the order of SR-OSjveto, SR-2jets, SR- $m_{\mathrm{T} 2}$ [18]. Values are in units of GeV.

\begin{tabular}{lccc}
\hline Selection & top & $W W$ & $Z+X$ \\
\hline$m_{\ell \ell}$ & $Z$-veto & $Z$-veto & $Z$-window \\
signal jets & $\geq 2$ & $=0$ & $=0, \geq 2, \geq 0$ \\
signal b-jets & $\geq 1$ & $=0$ & $\geq 0,=0, \geq 0$ \\
$E_{\mathrm{T}}^{\text {miss,rel. }}$ & $>100,50,40$ & $70-100$ & $>100,50,40$ \\
other & - & - &,$- m_{\mathrm{CT}}$-veto, - \\
\hline
\end{tabular}

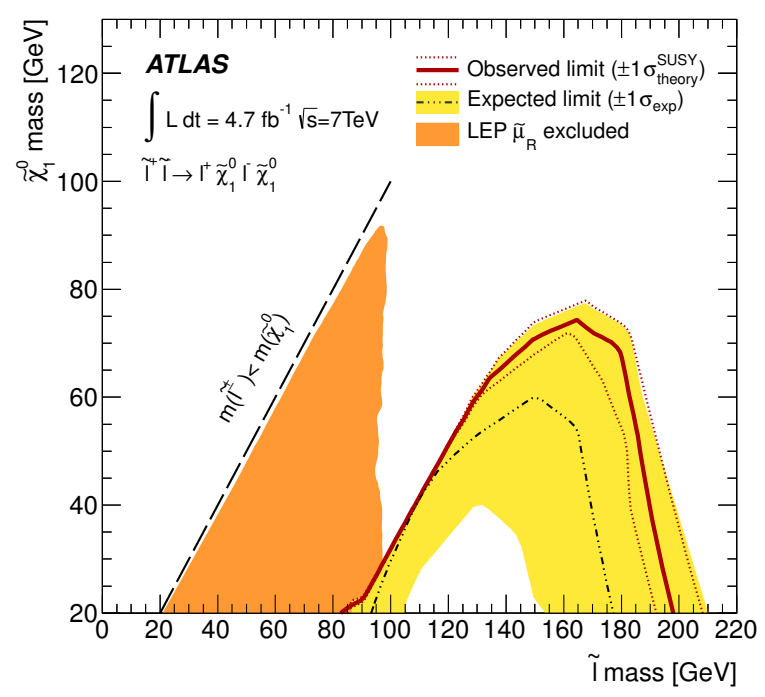

Figure 1. Observed and expected 95\% CL limit contours for $\tilde{\ell}^{ \pm}$pair production in the direct slepton pMSSM. The expected and observed limits are calculated without signal cross-section uncertainty taken into account. The yellow band is the $\pm 1 \sigma$ experimental uncertainty on the expected limit (black dashed line). The red dotted band is the $\pm 1 \sigma$ signal theory uncertainty on the observed limit (red solid line) [18]. Illustrated also is the LEP limit [28] on the mass of the right-handed smuon, $\tilde{\mu}_{R}$.

between 85 and $195 \mathrm{GeV}$ are excluded for a $20 \mathrm{GeV}$ neutralino. Figure 2 shows the $\tilde{\chi}_{1}^{\mp}$ pair production exclusion region in the simplified model. Chargino masses between 110 and $340 \mathrm{GeV}$ are excluded for a $10 \mathrm{GeV}$ neutralino.

The $4.7 \mathrm{fb}^{-1}$ two lepton $\tilde{\chi}_{2}^{0} \tilde{\chi}_{1}^{ \pm}$limits are not shown as they are superseded by the more recent $13 \mathrm{fb}^{-1}$ three lepton $\tilde{\chi}_{2}^{0} \tilde{\chi}_{1}^{ \pm}$limits, which are presented in Section 4.4.

\section{Three Lepton Analysis}

The three lepton analysis [29] uses $13 \mathrm{fb}^{-1}$ of protonproton collision data recorded by the ATLAS detector at $\sqrt{s}=8 \mathrm{TeV}$. The event selection, signal regions, background evaluation method and results are presented.

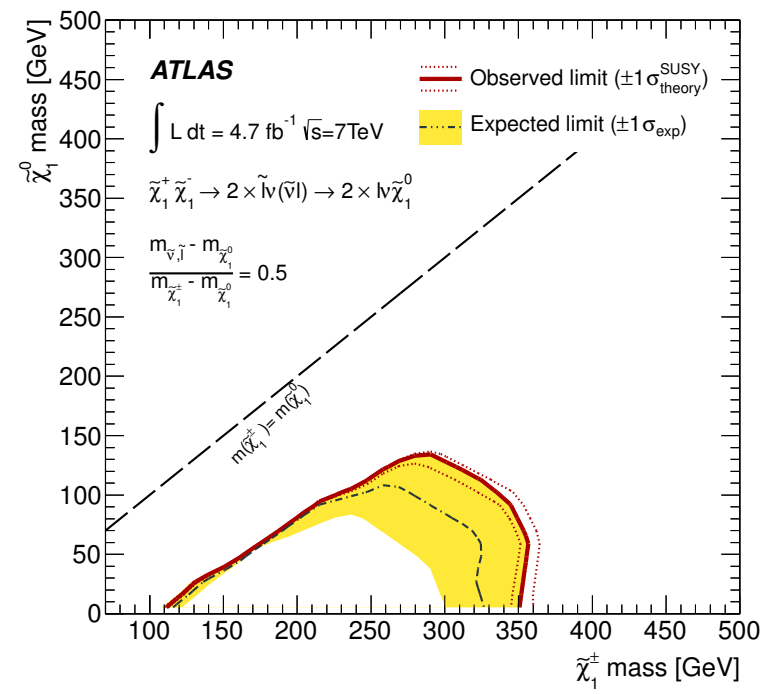

Figure 2. Observed and expected 95\% CL limit contours for $\tilde{\chi}_{1}^{ \pm}$ pair production in the simplified model scenario with intermediate sleptons [18]. The color coding is the same as in Figure 1.

\subsection{Event Selection}

Events are selected using an inclusive set of double-lepton triggers. Each reconstructed triggering lepton must be matched to an online lepton. The jet, electron and muon candidate definitions as well as the $E_{\mathrm{T}}^{\text {miss }}$ definition are the same as in Section 3.1. A subset of the object candidates are selected as signal objects. Signal jets must have $|\eta|<2.5$ and a JVF $>0.5$. Signal electrons and muons are required to be isolated, and the electrons must pass the "tight" selection criteria.

\subsection{Signal Regions}

The three lepton analysis targets $\tilde{\chi}_{2}^{0} \tilde{\chi}_{1}^{ \pm}$production, and uses three SRs corresponding to various $\tilde{\chi}_{2}^{0}$ decay modes. Their definitions are shown in Table 4. SR1a and SR1b target $\tilde{\chi}_{2}^{0}$ decays that proceed via sleptons and off-shell $Z$ bosons, while SR2 targets on-shell $Z$ bosons. Common to all these decay modes is the presence of a pair of same-flavor opposite-sign (SFOS) leptons in the final state. All SRs require exactly three leptons, including an SFOS pair with $m_{\mathrm{SFOS}}>12 \mathrm{GeV}$. The transverse mass is defined as $m_{\mathrm{T}}=\sqrt{2 \cdot E_{\mathrm{T}}^{\text {miss }} \cdot p_{\mathrm{T}}^{\ell} \cdot\left(1-\cos \Delta \phi_{\ell, E_{\mathrm{T}}^{\text {miss }}}\right)}$ where $\ell$ denotes the lepton that is not part of the pair forming the best Z-candidate. SR1b is a subset of SR1a, designed to increase sensitivity to models where the mass splitting between $\tilde{\chi}_{1}^{0}$ and the other gauginos is large.

\subsection{Background Evaluation}

As in the two lepton analysis, the fake background is obtained using the matrix method. The main real background comes from $W Z$, and is estimated using a refined version 
Table 4. Three lepton signal region definitions [29].

\begin{tabular}{|c|c|c|c|}
\hline Selection & SR1a & SR1b & SR2 \\
\hline Targeted $\tilde{\chi}_{2}^{0}$ decay & \multicolumn{2}{|c|}{$\tilde{\ell}^{(*)}$ or $Z^{*}$} & on-shell $Z$ \\
\hline$\left|m_{\mathrm{SFOS}}-m_{Z}\right|$ & \multicolumn{2}{|c|}{$>10 \mathrm{GeV}$} & $<10 \mathrm{GeV}$ \\
\hline Number of $b$-jets & \multirow{2}{*}{\multicolumn{2}{|c|}{0}} & any \\
\hline$E_{\mathrm{T}}^{\mathrm{miss}}$ & $>75 \mathrm{GeV}$ & & $>120 \mathrm{GeV}$ \\
\hline$m_{\mathrm{T}}^{1}$ & any & $>110 \mathrm{GeV}$ & $>110 \mathrm{GeV}$ \\
\hline$p_{\mathrm{T}}$ of leptons & $>10 \mathrm{GeV}$ & $>30 \mathrm{GeV}$ & $>10 \mathrm{GeV}$ \\
\hline
\end{tabular}

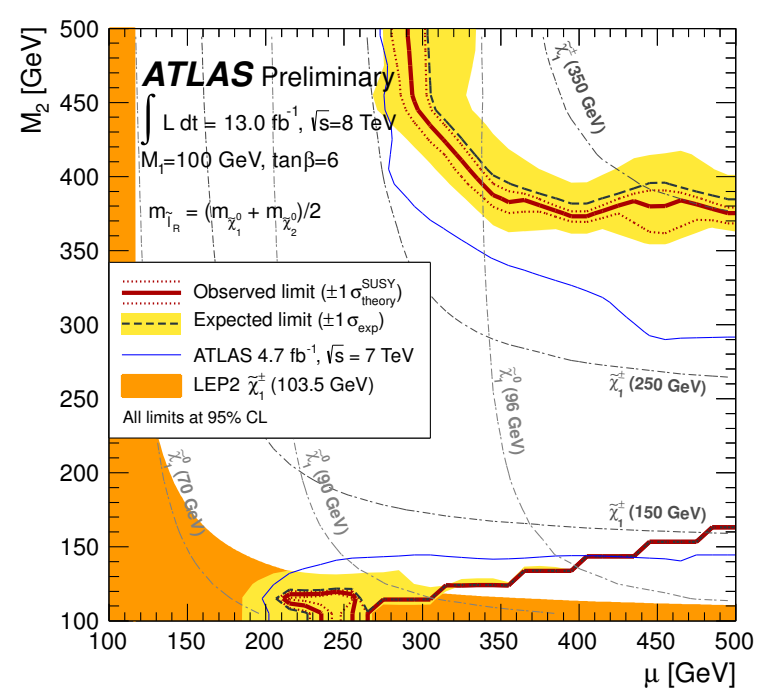

Figure 3. Observed and expected $95 \%$ CL limit contours for $\tilde{\chi}_{2}^{0} \tilde{\chi}_{1}^{ \pm}$production in the pMSSM for $M_{1}=100 \mathrm{GeV}$. The expected and observed limits are calculated without signal cross-section uncertainty taken into account. The yellow band is the $\pm 1 \sigma$ experimental uncertainty on the expected limit (black dashed line). The red dotted band is the $\pm 1 \sigma$ signal theory uncertainty on the observed limit (red solid line) [29]. The blue lines correspond to the $7 \mathrm{TeV}$ limits from the combination of the two lepton and three lepton analyses [30]. The LEP2 limit corresponds to the limit on the $\tilde{\chi}_{1}^{ \pm}$mass in [31] as transposed to this pMSSM plane.

of the method described in Section 3.3. This method includes a $W Z$ normalization factor that takes into account differences between data and MC in the $\mathrm{CR}$, as well as a non-negative signal contribution in the SR. These two parameters are kept floating and fitted simultaneously in the CR and SR to set limits on the cross section for new physics while correctly propagating the uncertainty on the normalization. The $W Z \mathrm{CR}$ requirements are: exactly three leptons with $p_{\mathrm{T}}>20 \mathrm{GeV}$, one SFOS lepton pair, a $Z$ candidate, $50<E_{\mathrm{T}}^{\text {miss }}<75 \mathrm{GeV}$, a $b$-veto, and $50<m_{\mathrm{T}}<$ $110 \mathrm{GeV}$. Remaining backgrounds are taken from $\mathrm{MC}$.

\subsection{Results}

Good agreement between data and Standard Model expectation is observed in all SRs. Upper limits on the production cross-section times acceptance times efficiency $(\sigma \times \varepsilon \times A)$ of new physics are set at the $95 \%$ confidence level using the modified frequentist $\mathrm{CL}_{\mathrm{s}}$ prescription.

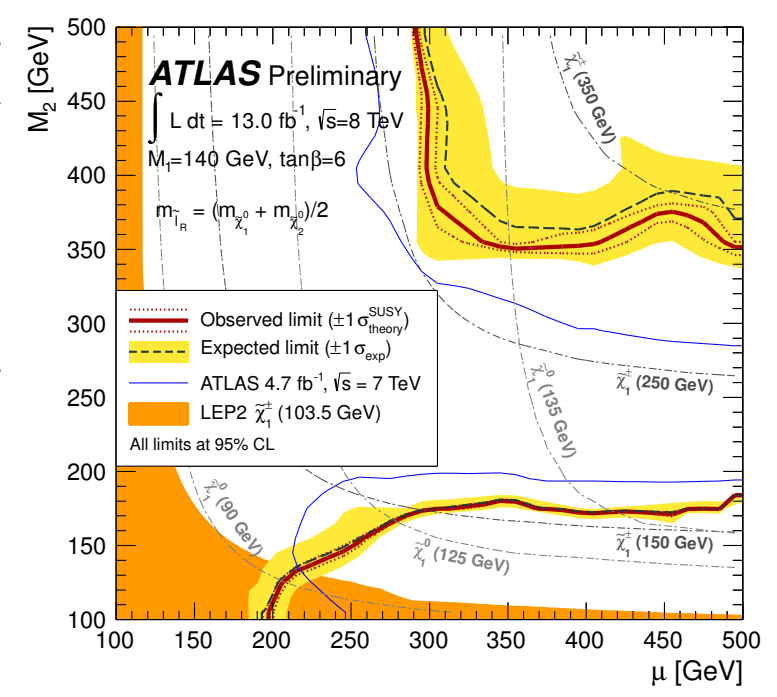

Figure 4. Observed and expected 95\% CL limit contours for $\tilde{\chi}_{2}^{0} \tilde{\chi}_{1}^{ \pm}$production in the pMSSM for $M_{1}=140 \mathrm{GeV}$ [29]. The color coding is the same as in Figure 3.

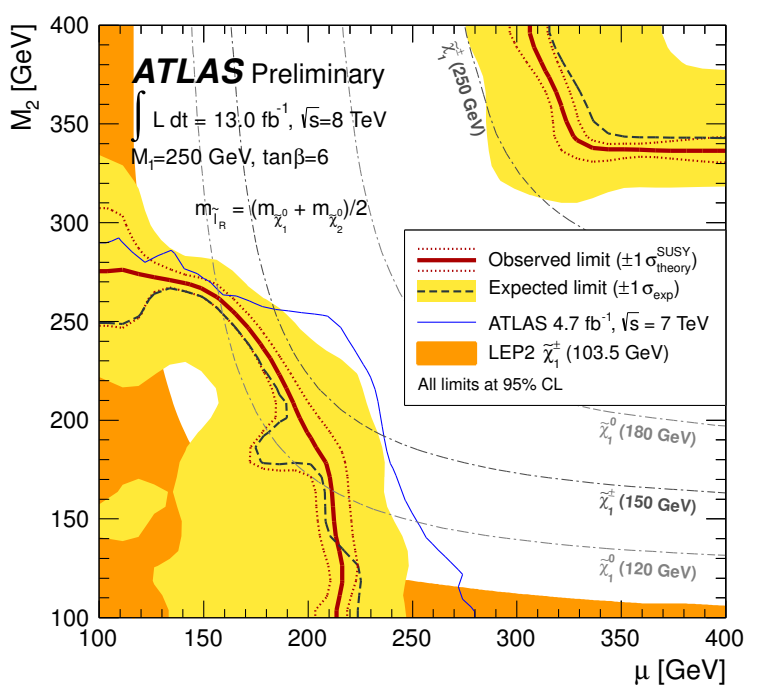

Figure 5. Observed and expected 95\% CL limit contours for $\tilde{\chi}_{2}^{0} \tilde{\chi}_{1}^{ \pm}$production in the pMSSM for $M_{1}=250 \mathrm{GeV}$ [29]. The color coding is the same as in Figure 3.

Figure 3, 4 and 5 shows the $\tilde{\chi}_{2}^{0} \tilde{\chi}_{1}^{ \pm}$production exclusion region in the pMSSM for $M_{1}=100 \mathrm{GeV}, M_{1}=140 \mathrm{GeV}$ and $M_{1}=250 \mathrm{GeV}$, respectively. Figure 6 and 7 show the $\tilde{\chi}_{2}^{0} \tilde{\chi}_{1}^{ \pm}$exclusion region in the simplified model scenarios with and without intermediate sleptons. Chargino masses are excluded up to $580 \mathrm{GeV}$ and $300 \mathrm{GeV}$, respectively.

\section{Summary}

A search for direct production of supersymmetric gauginos and sleptons in final states with leptons has been presented. The results are interpreted in the pMSSM and simplified models. No significant excess above the Standard Model expectation has been observed. In the simpli- 


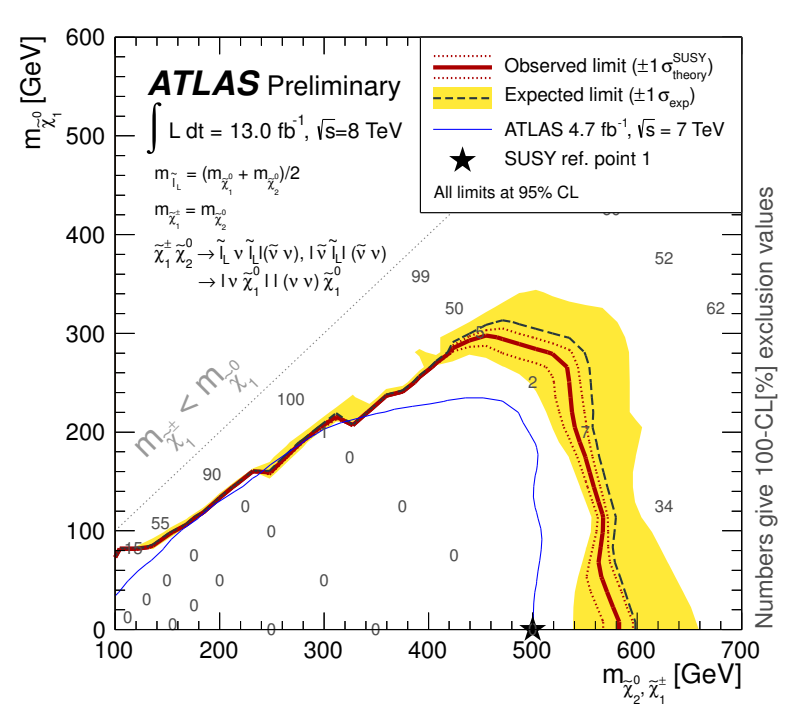

Figure 6. Observed and expected 95\% CL limit contours for $\tilde{\chi}_{2}^{0} \tilde{\chi}_{1}^{ \pm}$production in the simplified model scenario with intermediate sleptons. The 100-CL[\%] value is shown for each of the simulated model points [29]. The blue line corresponds to the 7 $\mathrm{TeV}$ limits from the three lepton analysis [30]. Remaining color coding is the same as in Figure 3.

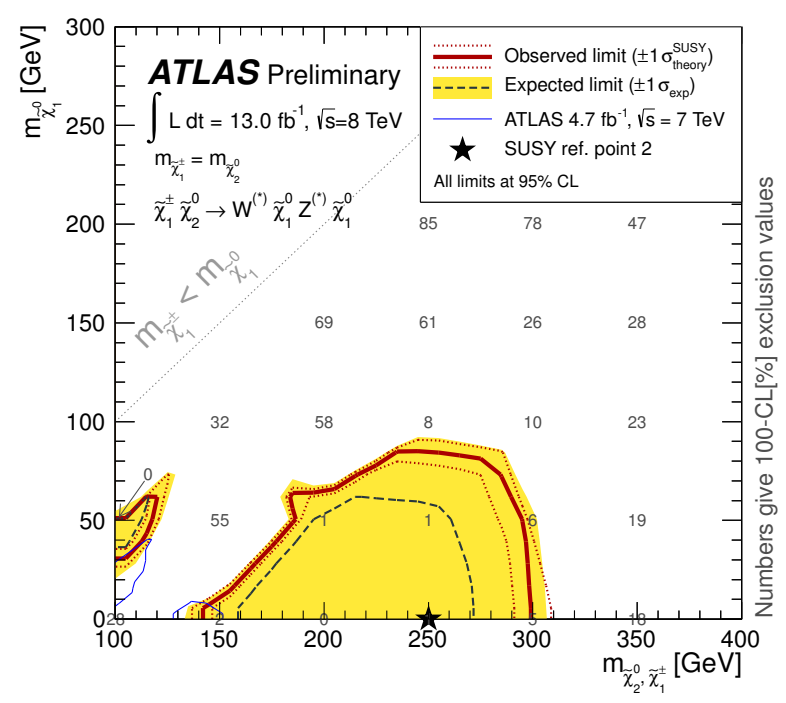

Figure 7. Observed and expected 95\% CL limit contours for $\tilde{\chi}_{2}^{0} \tilde{\chi}_{1}^{ \pm}$production in the simplified model scenario without intermediate sleptons. The 100-CL[\%] value is shown for each of the simulated model points [29]. The blue line corresponds to the 7 $\mathrm{TeV}$ limits from the three lepton analysis [30]. Remaining color coding is the same as in Figure 3.

fied model scenarios with and without intermediate sleptons, chargino masses are excluded up to $580 \mathrm{GeV}$ and $300 \mathrm{GeV}$, respectively.

\section{References}

[1] Miyazawa, H., Prog. Theor. Phys. 36 (6), 1266-1276 (1966)

[2] Ramond, P., Phys. Rev. D3, 2415-2418 (1971)

[3] Golfand, Y. A. et al., JETP Lett. 13, 323-326 (1971)

[4] Neveu, A. and Schwarz, J. H., Nucl. Phys. B31, 86112 (1971)

[5] Neveu, A. and Schwarz, J. H., Phys. Rev. D4, 11091111 (1971)

[6] Gervais, J. L. and Sakita, B., Nucl. Phys. B34, 632639 (1971)

[7] Volkov, D. V. and Akulov, V. P., Phys. Lett. B46, 109110 (1973)

[8] Wess, J. and Zumino, B., Phys. Lett. B49, 52 (1974)

[9] Wess, J. and Zumino, B., Nucl. Phys. B70, 39-50 (1974)

[10] Fayet, P., Phys. Lett. B64, 159 (1976)

[11] Fayet, P., Phys. Lett. B69, 39-50 (1977)

[12] Farrar, G. R. and Fayet, P., Phys. Lett. B76, 575-579 (1978)

[13] Fayet, P., Phys. Lett. B84, 416 (1979)

[14] Dimopoulos, S. and Georgi, H., Nucl. Phys. B193, 150 (1981)

[15] ATLAS Collaboration, JINST 3, S08003 (2008)

[16] Djouadi, A. et al., Comput. Phys. Commun. 176, 426-455, (2007)

[17] Alwall, J. et al., Phys. Rev. D79, 075020, (2009)

[18] ATLAS Collaboration, arXiv:1208.2884 [hep-ex]

[19] Cacciari, M., Salam, G. P. and Soyez, G., JHEP 04, 063 (2008)

[20] ATLAS Collaboration, JHEP 12, 060 (2010)

[21] Barr, A., Lester, C. and Stephens, P., J.Phys.G G29, 2343-2363 (2003)

[22] Lester, C. G. and Summers, D. J., Phys. Lett. B463, 99-103 (1999)

[23] Tovey, D. R., JHEP 04, 034 (2008)

[24] Polesello, G. and Tovey, D. R., JHEP 03, 030 (2010)

[25] ATLAS Collaboration, Eur. Phys. J. C71, 1577 (2011)

[26] ATLAS Collaboration, JHEP 04, 069 (2012)

[27] Read, A. L., J. Phys. G G28, 2693-2704 (2002)

[28] LEP SUSY Working Group (ALEPH, DELPHI, L3, OPAL), Notes LEPSUSYWG/01-03.1 and 04-01.1

[29] ATLAS Collaboration, ATLAS-COM-CONF-2012192, https://cds.cern.ch/record/1493493

[30] ATLAS Collaboration, arXiv:1208.3144 [hep-ex]

[31] Nakamura, K. and Particle Data Group, J. Phys. G: Nucl. Part. Phys. 37, 075021 (2010) 